\title{
TRANSPORT THROUGH ASYMMETRIC TWO-LEAD JUNCTIONS OF LUTTINGER LIQUID WIRES
}

\author{
D.N. Aristov $\sqrt{a b l c}$ and P. Wölfle $b \mid \mathrm{b}$ \\ ${ }^{a}$ Petersburg Nuclear Physics Institute, Gatchina 188300, Russia \\ ${ }^{\mathrm{b}}$ Institute for Nanotechnology, Karlsruhe Institute of Technology, 76021 Karlsruhe, Germany \\ E-mail: peter.woelfle@kit.edu \\ ${ }^{\mathrm{c}}$ Department of Physics, St.Petersburg State University, Ulianovskaya 1, St.Petersburg 198504, Russia \\ ${ }^{\mathrm{d}}$ Institute for Condensed Matter Theory, and Center for Functional Nanostructures, Karlsruhe Institute of Technology, 76128 \\ Karlsruhe, Germany
}

Received 20 April 2012; accepted 7 June 2012

\begin{abstract}
We calculate the conductance of a system of two spinless Luttinger liquid wires with different interaction strengths $g_{1}, g_{2}$, connected through a short junction, within the scattering state formalism. Following earlier work we formulate the problem in current algebra language, and calculate the scale dependent contribution to the conductance in perturbation theory keeping the leading universal contributions to all orders in the interaction strength. From that we derive a renormalization group (RG) equation for the conductance. The analytical solution of the RG-equation is discussed in dependence on $g_{1}, g_{2}$. The regions of stability of the two fixed points corresponding to conductance $G=0$ and $G=1$,respectively, are determined.
\end{abstract}

Keywords: Luttinger liquid wires, quantum transport, conductance, renormalization group theory

PACS: 71.10.Pm, 72.10.-d, 85.35.Be

\section{Introduction}

Charge or spin transport in three-dimensional metallic systems may be described in terms of Landau quasiparticles. In strictly one-dimensional quantum wires quasi-particles are found to be unstable on account of the interaction between electrons. An important part of that physics is captured by the exactly solvable Tomonaga-Luttinger liquid (TLL) model [1, 2]. As is well known, transport through junctions of TLL wires is strongly affected by the interaction in the wires, in some cases leading to a complete blocking of transmission. The latter behavior can be traced back to the formation of Friedel oscillations of the charge density around the barrier, leading to an infinitely extended effective barrier potential in the limit of low energy. The suitable language to describe this situation is the renormalization group (RG) method, allowing to calculate the conductance as a function of (length or energy) scale. Quite generally, the transport behavior at low energy/temperature is dominated by only a few fixed points of the RG flow. In the neighborhood of these fixed points the conductance is found to obey powerlaw behavior as a function of temperature $T$ for the infinite system or as a function of system length $L$ at zero temperature. The problem of the two-wire junction has been studied first in the pioneering works [3, 4], using the method of bosonization. Later, their results have been confirmed by many other authors, see [5] and references therein. For special values of the interaction exact results have been obtained [6, 7].

A purely fermionic formulation of the problem has been introduced in the limit of weak interaction by Yue, Glazman and Matveev [8]. We have extended that theory to the regime of strong coupling by summing up an infinite series of terms in perturbation theory, identified as the leading and universal contributions [9]. The results obtained with our method for the two-lead junction as well as the three lead junction with time-reversal symmetry [10, 11] and with magnetic flux [12] are in agreement with exact theoretical results, where available. However, our results go beyond what has been obtained by other authors in various ways. The majority of the previous works considered the symmetric case of equal interaction strength in both half wires, with exception of the works [13, 14] discussed below.

In this paper we generalize our previous treatment [9] to the case of two wires with different interaction strength $g_{1}, g_{2}$. This includes the case of a barrier at 
the end of a TLL wire, for which one of the interaction parameters is zero, e. g. $g_{2}=0$. As before, we confine our considerations to spinless fermions.

\section{The model}

To illustrate the system we are interested in, we first consider a tight binding Hamiltonian $\mathcal{H}_{t b}$ of free spinless fermions describing two quantum wires connected at a single junction by tunneling amplitudes:

$$
\mathcal{H}_{t b}=\left[\sum_{j=1}^{2} \sum_{n=0}^{N} t_{0} c_{j, n}^{+} c_{j, n+1}+t_{b} c_{2,0}^{+} c_{1,0}\right]+\text { H.c. }
$$

Here $c_{j, n}^{+}$creates a fermion in wire $j$ at site $n$, and $t_{b}$ is the tunneling amplitude connecting the sites $(j, n=$ $0)$ at the junction. The $2 \times 2 S$-matrix characterizing the scattering at this junction has the structure (up to overall phase factors in the individual wires)

$$
S=\left(\begin{array}{c}
r t \\
\widetilde{t} r
\end{array}\right)=\left(\begin{array}{cc}
\sin \theta & i \cos \theta e^{-i \phi} \\
i \cos \theta e^{i \phi} & \sin \theta .
\end{array}\right)
$$

We choose this parametrization in terms of the transmission and reflection amplitudes $t, r$, since it is readily generalizable to the case of multi-wire junctions ( $n$ wires, $n>2$ ). The above form of the $S$-matrix is completely general (up to irrelevant phase factors) and, in fact, defines our model. Passing to the continuum limit, linearizing the spectrum at the Fermi energy and adding forward scattering interaction of strength $g_{j}$ in wire $j$, we may write the TLL Hamiltonian $\mathcal{H}$ in the representation of incoming and outgoing waves as

$$
\begin{aligned}
& \mathcal{H}=\int_{-\infty}^{0} d x \sum_{j=1}^{2}\left[H_{j}^{0}+H_{j}^{i n t} \Theta(-L<x<-a)\right], \\
& H_{j}^{0}=v_{F} \psi_{j, \text { in }}^{\dagger} i \nabla \psi_{j, \text { in }}-v_{F} \psi_{j, \text { out }}^{\dagger} i \nabla \psi_{j, \text { out }}, \\
& H_{j}^{\text {int }}=2 \pi v_{F} g_{j} \psi_{j, \text { in }}^{\dagger} \psi_{j, \text { in }} \psi_{j, \text { out }}^{\dagger} \psi_{j, \text { out }} .
\end{aligned}
$$

We put $v_{F}=1$ from now on. The range of the interaction lies within the interval $(a, L)$, where $a>0$ serves as a ultraviolet cutoff and separates the domains of interaction and potential scattering on the junction; non-interacting leads correspond to large $|x|$ beyond $L$. In terms of the doublet of incoming fermions $\Psi=\left(\psi_{1, i n}, \psi_{2, \text { in }}\right)$ the outgoing fermion operators may be expressed with the aid of the $S$-matrix as $\Psi(x)=S \cdot \Psi(-x)$. We express the interaction term of the Hamiltonian in terms of density operators $\widehat{\rho}_{j, \text { in }}=\psi_{j, \text { in }}^{\dagger} \psi_{j, \text { in }}=\Psi^{+} \rho_{j} \Psi=\widehat{\rho}_{j}$, and $\widehat{\rho}_{j, \text { out }}=$ $\psi_{j, \text { out }}^{\dagger} \psi_{j, \text { out }}=\Psi^{+} \widetilde{\rho}_{j} \Psi=\widehat{\widetilde{\rho}}_{j}$, where $\widetilde{\rho}_{j}=S^{+} \cdot \rho_{j} \cdot S$, as

$$
H_{j}^{i n t}=2 \pi g_{1} \widehat{\rho}_{1} \widehat{\widetilde{\rho}}_{1}+2 \pi g_{2} \widehat{\rho}_{2} \widehat{\widetilde{\rho}}_{2} .
$$

The matrices are given by $\left(\rho_{j}\right)_{\alpha \beta}=\delta_{\alpha \beta} \delta_{\alpha j}$ and $\left(\widetilde{\rho}_{j}\right)_{\alpha \beta}=S_{\alpha j}^{+} S_{j \beta}$. A convenient representation of $2 \times 2$-matrices is in terms of Pauli matrices $\sigma_{j}, j=$ $1,2,3$, the generators of $S U(2)$ (see [9]). Notice that the interaction operator only involves $\sigma_{3}$ (besides the unit operator $\left.\left(\sigma_{0}\right)_{\alpha \beta}=\delta_{\alpha \beta}\right)$. We note $\operatorname{Tr}\left(\sigma_{j}\right)=0, \operatorname{Tr}\left(\sigma_{j} \sigma_{k}\right)=2 \delta_{j k}, j=0,1,2,3$. Defining a two-component vector $\mathbf{s}=\left(\sigma_{3}, \sigma_{0}\right)$, we have $\rho_{j}=\sqrt{1 / 2} \sum_{\mu} R_{j \mu} s_{\mu}$, where the $2 \times 2$ matrix $\mathbf{R}$ has the properties $\mathbf{R}^{-1}=\mathbf{R}^{T}$, $\operatorname{det} \mathbf{R}=1$, and $R_{11}=R_{12}=-R_{21}=R_{22}=1 / \sqrt{2}$. The outgoing amplitudes will be expressed in terms of $\widetilde{\sigma}_{j}=$ $S^{+} \cdot \sigma_{j} \cdot S$. With the aid of the $\sigma_{j}$ the $S$-matrix may be parametrized by three angular (Euler) variables (see [9]), $S=e^{i \alpha_{1} \sigma_{3} / 2} e^{i \alpha_{2} \sigma_{1} / 2} e^{i \alpha_{3} \sigma_{3} / 2}$. For the case under consideration only two of these, $\theta, \phi$, are relevant: $S=e^{-i \phi \sigma_{3} / 2} e^{i(\pi-2 \theta) \sigma_{1} / 2} e^{i \phi \sigma_{3} / 2}$. The corresponding elements of the $S$-matrix have been given in Eq. (2).

\section{Parametrization of conductance matrix}

We may define a $2 \times 2$ matrix of conductances $G_{j k}$ relating the current $I_{j}$ in lead $j$ (flowing towards the junction) to the electrical potential $V_{k}$ in lead $k$ : $I_{j}=\sum_{k} G_{j k} V_{k}$. It follows from the conservation of charge that $\sum_{j} G_{j k}=0$ and from invariance under a shift of the zero of energy that $\sum_{k} G_{j k}=0$. Therefore, the conductance matrix has only one independent element $G=(1-a) / 2$, which relates the net current $I=\frac{1}{2}\left(I_{1}-I_{2}\right)$ to the bias voltage $V=\left(V_{1}-V_{2}\right)$, $I=G V$. We note the relation $G=\frac{1}{2}\left(\mathbf{R}^{T} \mathbf{G R}\right)_{11}$, while all other elements of $\mathbf{R}^{T} \mathbf{G R}$ are zero.

In the linear response regime the conductances are related to the $S$-matrix by $G_{j k}=\delta_{j k}-\operatorname{Tr}\left(\tilde{\rho}_{j}^{r} \rho_{k}\right)=$ $\delta_{j k}-\left|S_{j k}^{r}\right|^{2}$, where the label $r$ indicates that the quantity is fully renormalized by interactions. Defining $Y_{j k}=$ $\operatorname{Tr}\left(\tilde{\rho}_{j}^{r} \rho_{k}\right)$ and using the above relation of $\tilde{\rho}_{j}^{r}$ and $\tilde{s}_{\mu}^{r}$, we see that the conductance components may be expressed in terms of $\bar{Y}_{\mu \nu}=\frac{1}{2} \operatorname{Tr}\left(\tilde{s}_{\mu}^{r} s_{\nu}\right)$ as $Y_{j k}=\left(\mathbf{R} \overline{\mathbf{Y}} \mathbf{R}^{T}\right)_{j k}$. Here and in the following bold faced quantities marked with overbar are matrices in the transformed space (indices $\mu, \nu)$. It follows from the analysis of the transformed matrix $\mathbf{R}^{T} \mathbf{G R}$ given above that the matrix $\overline{\mathbf{Y}}$ has block structure, the nonzero elements being given by the conductance parameter introduced above and by 
(a)

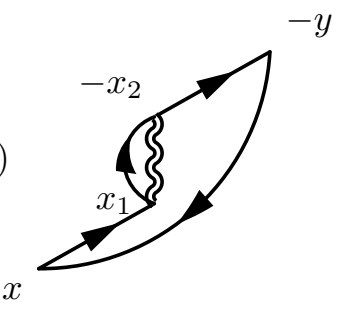

(b)

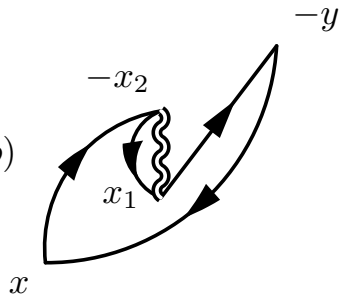

(c)

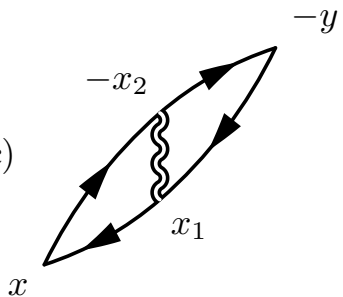

Fig. 1. Feynman diagrams depicting the corrections to conductance. Two first diagrams, (a) and (b), correspond to Eq. (8), the third diagram ("vertex correction") vanishes in the static limit, external $\Omega \rightarrow 0$.

unity, $\bar{Y}_{11}=a, \bar{Y}_{22}=1, \bar{Y}_{12}=\bar{Y}_{21}=0$. From the above relations we see that the parameter $a$ may be expressed by the angle $\theta$ in the above parametrization of the S-matrix as $a=-\cos 2 \theta$. We find therefore that $a$ is confined within the region $a \in[-1,1]$.

\section{Perturbation theory}

We now calculate the conductance in perturbation theory in the interaction. In first order we have to evaluate the diagrams depicted in Fig. 1. Here solid lines denote Green's functions in position-energy representation ( $\omega_{n}$ are fermionic Matsubara frequencies), $\mathcal{G}\left(x ; \omega_{n}\right)=-i \operatorname{sign}\left(\omega_{n}\right) \theta\left(x \omega_{n}\right) e^{-x \omega_{n}}$. The double wavy lines denote the interaction operator, which in the lowest order is given by the matrix $\bar{g}_{\mu \nu}=\left(\mathbf{R}^{T} \mathbf{g R}\right)_{\mu \nu}$, with $g_{j k}=\delta_{j k} g_{j}$, in the transformed or initial representations, respectively. At the ends of the interaction lines operators $\sigma_{3}$ and $\widetilde{\sigma}_{3}$ are attached depending on whether $x<0$ or $x>0$. As a result, one finds in lowest order in the interaction

$$
\begin{aligned}
\bar{Y}_{\mu \nu}^{(1)} & =-\frac{1}{2} \operatorname{Tr}\left(\widehat{\bar{W}}_{\mu \nu} \widehat{\bar{W}}_{\mu^{\prime} \nu^{\prime}}\right) \bar{g}_{\nu^{\prime} \mu^{\prime}} \Lambda \\
& =-\frac{1}{2} \delta_{\mu 1} \delta_{\nu 1}\left(a^{2}-1\right)\left(g_{1}+g_{2}\right) \Lambda .
\end{aligned}
$$

Here the $\widehat{W}_{\mu \nu}=\left(\mathbf{R}^{T} \widehat{\mathbf{W}} \mathbf{R}\right)_{\mu \nu}=\frac{1}{2}\left[s_{\mu}, \widetilde{s}_{\nu}\right]$, and $\widehat{W}_{j k}=\left[\rho_{j}, \widetilde{\rho}_{k}\right]$, are $2 \times 2$ matrices for each pair of $\mu \nu$ (or $j k$ ) and the trace operation $\operatorname{Tr}$ is defined with respect to that matrix space, whereas the $\bar{g}_{\mu \nu}=$ $\left(\mathbf{R}^{T} \mathbf{g R}\right)_{\mu \nu}$, with $g_{j k}=\delta_{j k} g_{j}$ are scalars. Notice that only one matrix element, $\widehat{W}_{11}$, is nonzero.

We may extend this analysis into the strong coupling regime by summing up infinite classes of contributions in perturbation theory. It has been found in [9] that the diagrams shown in Figs. 1 and 2 provide the leading terms in the neighborhood of the fixed points. Their contribution is universal in contrast to other higher order terms (see below). These may be interpreted as a renormalization of the bare interaction, $2 \pi g_{k l} \delta(x-y) \rightarrow L_{k l}\left(x, y ; \omega_{n}\right)$. The contribution to conductance stemming from the first two diagrams (a) and (b) in Fig. 1 in this ladder approximation is given by

$$
\begin{aligned}
& G^{(a+b)}=-\frac{1}{4} T^{2} \sum_{\epsilon, \omega} \int_{a}^{L} d x_{1} d x_{2} \int_{L}^{\infty} d y \\
& \times \bar{L}_{11}\left(x_{1}, x_{2} ; \omega\right) \mathcal{G}(y+x ; \epsilon+\Omega)\left[\mathcal{G}\left(x_{1}-x ; \epsilon\right)\right. \\
& \times \mathcal{G}\left(-x_{1}-x_{2} ; \epsilon-\omega\right) \mathcal{G}\left(x_{2}-y ; \epsilon\right)+\cos 4 \theta \\
& \left.\times \mathcal{G}\left(-x_{2}-x ; \epsilon\right) \mathcal{G}\left(x_{1}+x_{2} ; \epsilon+\omega\right) \mathcal{G}\left(-x_{1}-y ; \epsilon\right)\right]
\end{aligned}
$$

Here only the $(1,1)$ element of $\overline{\mathbf{L}}$ enters, corresponding to the fact that operators $\sigma_{3}$ and $\widetilde{\sigma}_{3}$ are attached to the ends of the renormalized interaction line. The factor $\cos 4 \theta$ appears as $\operatorname{Tr}\left(\sigma_{3} \widetilde{\sigma}_{3} \sigma_{3} \widetilde{\sigma}_{3}\right) / 2$. In the limit of zero temperature we may convert the summation over Matsubara frequencies to an integration along the imaginary axis. Another contribution to $G$ is obtained by reverting the arrows on the fermion lines in Fig. 11(a), Fig. 1. b), and doubles the above result. Performing the integrations on $\epsilon$ and on $y$ from $L$ to $\infty$ and taking the limit $\Omega \rightarrow 0$ we find

$$
\begin{aligned}
& G^{(L)}=\left(1-a^{2}\right) \\
& \times \int d x_{1} d x_{2} \frac{d \omega}{2 \pi} \bar{L}_{11}\left(x_{1}, x_{2} ; \omega\right) \theta(\omega) e^{-\omega\left(x_{1}+x_{2}\right)},
\end{aligned}
$$

It is useful to first calculate $\mathbf{L}$ in the initial representation, where the interaction matrix $\mathbf{g}$ is diagonal, but the matrix $\mathbf{Y}$ is nondiagonal. Then $\mathbf{L}$ is found to satisfy the following linear integral equation for $\omega>0$ (Ref. [9], 
note that the definitions of $\mathbf{Y}$ and for the strength of interaction there are different):

$$
\begin{aligned}
& \left(\begin{array}{c}
\mathbf{L}(x, y ; \omega) \\
\mathbf{L}_{2}(x, y ; \omega)
\end{array}\right)=2 \pi \mathbf{g} \delta(x-y)\left(\begin{array}{c}
1 \\
0
\end{array}\right)-2 \pi \int_{a}^{L} d z \\
& \times\left(\begin{array}{cc}
\mathbf{g Y} \Pi(x+z, \omega), \mathbf{g} \Pi(x-z, \omega) \\
\mathbf{g} \Pi(z-x, \omega), & 0
\end{array}\right)\left(\begin{array}{c}
\mathbf{L}(z, y ; \omega) \\
\mathbf{L}_{2}(z, y ; \omega)
\end{array}\right)
\end{aligned}
$$

with the fermionic loop $\Pi\left(x, \omega_{n}\right)=(2 \pi)^{-1}(\delta(x)-$ $\left.\left|\omega_{n}\right| \theta\left(x \omega_{n}\right) e^{-x \omega_{n}}\right)$. Expressing $\quad \mathbf{L}_{2}(x, y ; \omega) \quad$ via $\mathbf{L}(x, y ; \omega)$ we have

$$
\begin{aligned}
& \mathbf{L}(x, y ; \omega)=2 \pi \widetilde{\mathbf{g}} \delta(x-y)+\omega \widetilde{\mathbf{g}} \int_{a}^{L} d z \\
& \times\left[\left(\mathbf{Y}-\frac{1}{2} \mathbf{g}\right) e^{-\omega(x+z)}-\frac{1}{2} \mathbf{g} e^{-\omega|x-z|}\right] \mathbf{L}(z, y ; \omega),
\end{aligned}
$$

with $\widetilde{g}_{j}=g_{j} / d_{j}^{2}$ and $d_{j}=\sqrt{1-g_{j}^{2}}$. In order to solve this integral equation we first calculate a partial summation, defined by $\mathbf{C}(x, y ; \omega)=\lim _{Y \rightarrow 0} \mathbf{L}(x, y ; \omega)$.

Then $\mathbf{L}(x, y ; \omega)$ will be the solution of

$$
\begin{aligned}
& \mathbf{L}(x, y ; \omega)=\mathbf{C}(x, y ; \omega)+\frac{\omega}{2 \pi} \int_{a}^{L} d z_{1} d z_{2} \\
& \times \mathbf{C}\left(x, z_{1} ; \omega\right) \mathbf{Y} e^{-\omega\left(z_{1}+z_{2}\right)} \mathbf{L}\left(z_{2}, y ; \omega\right),
\end{aligned}
$$

and $\mathbf{C}(x, y ; \omega)$ satisfies the integral equation

$$
\begin{aligned}
& \mathbf{C}(x, y ; \omega)=2 \pi \widetilde{\mathbf{g}} \delta(x-y)-\frac{1}{2} \omega \widetilde{\mathbf{g} g} \int_{a}^{L} d z \\
& \times\left[e^{-\omega(x+z)}+e^{-\omega|x-z|}\right] \mathbf{C}(z, y ; \omega) .
\end{aligned}
$$

These integral equations are shown diagrammatically in Fig. 2,

We now define $\mathbf{C}(x, y ; \omega)=2 \pi \widetilde{\mathbf{g}} \delta(x-y)-$ $\mathbf{C}_{1}(x, y ; \omega)$, so that the inhomogeneity in the integral equation for $\mathbf{C}_{1}(x, y ; \omega)$ is differentiable

$$
\begin{aligned}
& \mathbf{C}_{1}(x, y ; \omega)=\pi \omega \widetilde{\mathbf{g}}^{2} \mathbf{g}\left[e^{-\omega(x+y)}+e^{-\omega|x-y|}\right] \\
& -\frac{1}{2} \omega \widetilde{\mathbf{g} g} \int_{a}^{L} d z\left[e^{-\omega(x+z)}+e^{-\omega|x-z|}\right] \mathbf{C}_{1}(z, y ; \omega),
\end{aligned}
$$

The integral equation for $\mathbf{C}_{1}(x, y ; \omega)$ may be converted into a second order differential equation
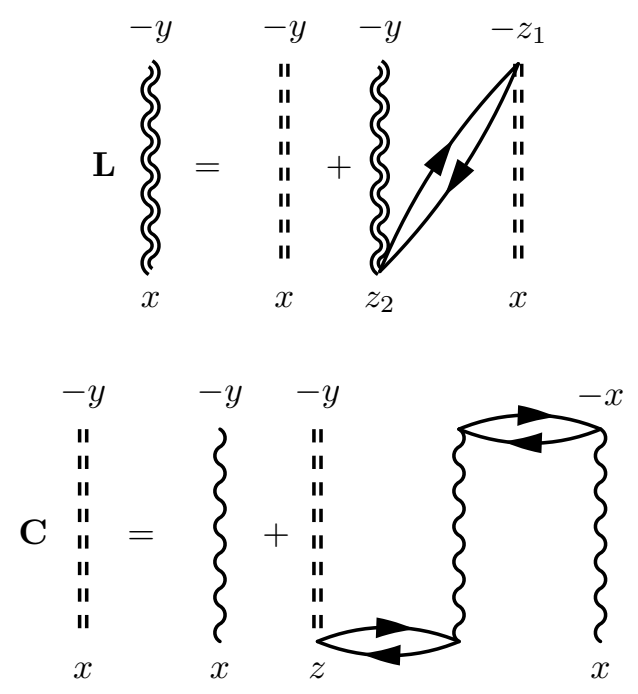

Fig. 2. Feynman diagrams depicting the integral equations for the renormalized interaction, Eqs. (9) and (10).

$$
\begin{aligned}
& {\left[\frac{\partial^{2}}{\partial x^{2}} \mathbf{1}-\omega^{2}(\mathbf{1}+\widetilde{\mathbf{g} g})\right] \mathbf{C}_{1}(x, y ; \omega)=} \\
& -2 \pi \omega^{2} \widetilde{\mathbf{g}}^{2} \mathbf{g} \delta(x-y),
\end{aligned}
$$

Since the matrix $\mathbf{g}$ is diagonal, $\mathbf{C}_{1}$ is diagonal and we have two uncoupled differential equations for the components $C_{1, j}, j=1,2$. Taking into account the boundedness of $C_{1, j}$, the general solution is given by $C_{1, j}(x, y ; \omega)=\pi \omega_{j} \widetilde{g}_{j} g_{j}^{2}\left(A_{j}(y) e^{-\omega_{j} x}+e^{-\omega_{j}|x-y|}\right)$, where $\omega_{j}=\omega / d_{j}$. It follows from the boundary conditions at $x=0$ that $A_{j}(y)=e^{-\omega_{j} y}$. The quantity $\mathbf{C}$ is thus a diagonal matrix given by

$$
\begin{aligned}
\mathbf{C}(x, y ; \omega)= & 2 \pi \mathbf{d}^{-2} \mathbf{g} \delta(x-y) \\
& -\pi \omega \mathbf{d}^{-3} \mathbf{g}^{3}\left[e^{-\omega(x+y) / \mathbf{d}}+e^{-\omega|x-y| / \mathbf{d}}\right]
\end{aligned}
$$

We now return to the integral equation for $\mathbf{L}(x, y ; \omega)$. Since the kernel is separable it is easily solved. We define the auxiliary matrix functions $\mathbf{U}(y ; \omega)=\int d x$ $\times e^{-\omega x} \mathbf{L}(x, y ; \omega), \mathbf{V}(y ; \omega)=\int d x e^{-\omega x} \mathbf{C}(x, y ; \omega)=$ $2 \pi \mathbf{d}^{-1} \mathbf{g} e^{-\omega y / \mathbf{d}}$ as well as the matrix $\mathbf{Q}^{-1}=\frac{\omega}{2 \pi} \int d x d y$ $\times e^{-\omega(x+y)} \mathbf{C}(x, y ; \omega)=\mathbf{g}(\mathbf{1}+\mathbf{d})^{-1}$. Multiplying the above integral equation $(9)$ by $e^{-\omega x}$ and integrating over $x$ we find $\mathbf{U}=\mathbf{V}+\mathbf{Q}^{-1} \mathbf{Y} \mathbf{U}=\left(\mathbf{1}-\mathbf{Q}^{-1} \mathbf{Y}\right)^{-1} \mathbf{V}$ and we finally get

$\mathbf{L}(x, y ; \omega)=\mathbf{C}(x, y ; \omega)$

$$
+\frac{\omega}{2 \pi} \mathbf{V}(x ; \omega) \mathbf{Y}\left(\mathbf{1}-\mathbf{Q}^{-1} \mathbf{Y}\right)^{-1} \mathbf{V}(y ; \omega) .
$$


This result is now substituted into the expression for the conductance. Performing the integration over $y$ first we get $\int d y e^{-\omega y} \mathbf{L}(x, y ; \omega)=\mathbf{V}(x ; \omega)[\mathbf{1}+\mathbf{Y}(\mathbf{1}-$ $\left.\left.\mathbf{Q}^{-1} \mathbf{Y}\right)^{-1} \mathbf{Q}^{-1}\right]=\mathbf{V}(x ; \omega)\left[\mathbf{1}-\mathbf{Y Q}^{-1}\right]^{-1}$. Next integrating over positive $\omega$ yields $\int(d \omega / 2 \pi) e^{-\omega x} \mathbf{V}(x ; \omega)$ $\times\left[\mathbf{1}-\mathbf{Y Q}^{-1}\right]^{-1}=(1 / x) \mathbf{g}(\mathbf{1}+\mathbf{d})^{-1}\left[\mathbf{1}-\mathbf{Y Q}^{-1}\right]^{-1}=$ $(1 / x)[\mathbf{Q}-\mathbf{Y}]^{-1}$. Finally, the integration over $x$ produces the scale dependent logarithm $\Lambda=\ln (L / a)$. The conductance in the ladder approximation is found after transforming to the rotated basis (quantities with overbar) and taking the $(1,1)$-element of the matrix,

$$
G^{(L)}=\frac{1}{2}(1-a)-\left(1-a^{2}\right)\left[(\overline{\mathbf{Q}}-\overline{\mathbf{Y}})^{-1}\right]_{11} \Lambda .
$$

Renormalization group equations. The renormalization of the conductances by the interaction is determined from the scale dependent contributions in perturbation theory. Differentiating these results with respect to $\Lambda$ (and then putting $\Lambda=0$ ) we find the RG equation for the quantity $a=1-2 G$ in the ladder approximation

$$
\frac{d a}{d \Lambda}=2\left(1-a^{2}\right)\left[(\overline{\mathbf{Q}}-\overline{\mathbf{Y}})^{-1}\right]_{11}
$$

Here $\overline{\mathbf{Y}}=\operatorname{diag}(a, 1)$ and $\overline{\mathbf{Q}}=\mathbf{R}^{T} \mathbf{Q R}$, such that $\bar{Q}_{11}=\bar{Q}_{22}=\bar{Q}_{+}=\left(q_{1}+q_{2}\right) / 2$ and $\bar{Q}_{12}=\bar{Q}_{21}=$ $\bar{Q}_{-}=\left(q_{1}-q_{2}\right) / 2$ where $q_{j}=\left(1+\sqrt{1-g_{j}^{2}}\right) / g_{j}=$ $\left(1+K_{j}\right) /\left(1-K_{j}\right)$, and $K_{j}=\sqrt{\left(1-g_{j}\right) /\left(1+g_{j}\right)}$ is the usual Luttinger liquid parameter for wire $j$. We define

$$
\gamma=\bar{Q}_{+}-\bar{Q}_{-}^{2} /\left(\bar{Q}_{+}-1\right)=\frac{K_{1}^{-1}+K_{2}^{-1}+2}{K_{1}^{-1}+K_{2}^{-1}-2},
$$

(note that $|\gamma|>1$ for any $K_{1,2}>0$ ), then the RGequation takes the explicit form

$$
\frac{d a}{d \Lambda}=2 \frac{a^{2}-1}{a-\gamma}
$$

$R G$ flow and conductance. The fixed points of the above RG equation are labelled $N$, at $a=1, G=0$, (complete separation of the wires) and $A$ at $a=-1$ , $G=1$ (ideal conductance through the junction). In order to discuss the stability and to calculate the conductance we rewrite the RG-equation in terms of the conductance

$$
\frac{d G}{d \Lambda}=-4 \frac{G(1-G)}{2 G-1+\gamma}=\beta(G) .
$$

Stability of a fixed point requires that the derivative of $\beta(G)$ at the fixed point is negative. At fixed point $N$ this translates into $\gamma-1>0$, or more explicitly, $q_{1}+q_{2}>2$, and in terms of the Luttinger parameters

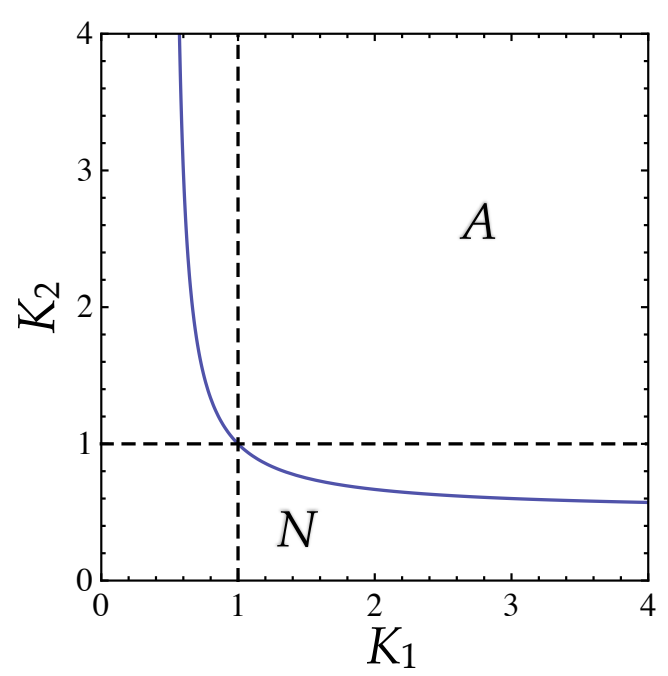

Fig. 3. Stability regions, labeled by the corresponding fixed point are shown in $K_{1}-K_{2}$ plane. The hyperbola separating the regions is shown by solid line, the non-interacting values $K_{1,2}=1$ are shown by dashed lines.

$K_{1}^{-1}+K_{2}^{-1}>2$ i. e. either both interactions should be repulsive, or one of them is attractive, but weak relative to the repulsive one. At fixed point $A$ the condition is $\gamma+1<0$, or $q_{1}+q_{2}<2$, and therefore $K_{1}^{-1}+K_{2}^{-1}<2$, meaning that the interaction is predominantly attractive, but a weaker repulsive interaction in one of the wires is possible. The line separating the stability regions in the $K_{1}-K_{2}$-plane is the hyperbola, $\left(K_{1}-\frac{1}{2}\right)\left(K_{2}-\frac{1}{2}\right)=\frac{1}{4}$, passing through the (no interaction) point $K_{1}=K_{2}=1$. This hyperbola and corresponding stability regions are shown in Fig. 3 .

The RG-equation may be integrated to give

$$
G^{1-\gamma}(1-G)^{1+\gamma}=c(L / a)^{4} \text {. }
$$

In the vicinity of fixed points $N$ and $A$, respectively, we then find the power laws

$$
\begin{aligned}
& G=c_{N}(a / L)^{4 /(\gamma-1)}, \quad \text { at } N, \\
& G=1-c_{A}(L / a)^{4 /(\gamma+1)}, \quad \text { at } A .
\end{aligned}
$$

Explicitly we have $4 /(\gamma-1)=\left(K_{1}^{-1}+K_{2}^{-1}-2\right)$, and $4 /(\gamma+1)=2\left(K_{1}+K_{2}-2 K_{1} K_{2}\right) /\left(K_{1}+K_{2}\right)$. At $K_{1}=K_{2}=K$ we recover the well-known [3, 4] exponents $2\left(K^{-1}-1\right), 2(1-K)$.

Of special interest is the case $g_{1}=g, g_{2}=0$, when we have $\gamma=2 q-1=(1+3 K) /(1-K)$ and the power laws are given by $G=c_{N}(L / a)^{-\left(K^{-1}-1\right)}$ at $N$ and $1-G=c_{A}(L / a)^{-2(K-1) /(K+1)}$ at $A$. We may compare these findings with results obtained in Ref. [13] for a non-symmetric position of an impurity in a TLL wire. When taking the impurity position sufficiently close to the interface between the TLL wire 
and a non-interacting lead, scaling exponents identical to the above ones were found in [13], as can be read off from Eqs. (10) and (13) there.

Thus we recover the correct exponents in the scaling law for the conductance. We obtained them by summation of the ladder sequence for the renormalized interaction in the presence of the junction. As was shown in [9], the first contributions to conductance beyond the ladder series appear in the third order of interaction and are of three-loop type (the two-loop RG contributions are absent). These latter terms do not contribute to above scaling exponents, but define the relation between the prefactors $c_{N}$ and $c_{A}$. The Eq. (15) implies that both $c_{N}$ and $c_{A}$ depend on the initial conditions, encoded in the amplitude $c$. It is easily seen, that the ladder approximation corresponds to the relation

$$
c_{N}^{1-\gamma} / c_{A}^{1+\gamma}=1
$$

It was shown [9] that the three-loop corrections change this ratio to values, different from unity and depending on the strength of interaction and regularization scheme, i. e. non-universal.

Our expression (12) shows that the boundary exponents depend only on the sum $K_{1}^{-1}+K_{2}^{-1}$. This is in precise agreement with Eq. (13) in Ref. [14], where the scaling exponent of the point contact between two chiral (Hall edge) states was derived. On the basis of this observation it was suggested there, that the combination of two chiral states, $K_{1}=1 / 3$ and $K_{2}=1$, is equivalent to the well-known problem of an impurity in a TLL wire with $K_{1}=K_{2}=1 / 2$, which can be fully solved [3]. We note here that the coincidence of the scaling exponents at two fixed points $N$ and $A$ might not necessarily mean the coincidence of the full scaling form for the conductance. The non-universal three-loop terms (which are not discussed in the standard bosonization approach) may be different in cases $K_{1}=1 / 3, K_{2}=1$ and $K_{1}=K_{2}=1 / 2$.

Conclusion. In this paper we employed a fermionic description of a general two-wire junction of two TLLwires to derive the renormalization group equation for the conductance, using the approach developed by us earlier [9]. We used an infinite summation of perturbation theory in the form of a ladder approximation, allowing for an analytical solution for arbitrary junction parameters and interactions in the wires. As demonstrated earlier the approximation is asymptotically exact in the vicinity of the fixed points. As in the well-studied case of a symmetric junction there exist two fixed points of the RG flow. Fixed point $N$ corresponds to a complete separation of the wires, i. e. the conductance vanishes. It is stable in a region of the $K_{1}-K_{2}-$ coupling constant plane which is predominantly repulsive, meaning that a weakly attractive component, say $K_{2}>1$ is permitted. At fixed point $A$ the conductance assumes its maximum value. It is stable in the complementary part of the coupling constant plane. The two stability regions in the $K_{1}-K_{2}-$ plane are separated by a hyperbolic boundary curve. The representation chosen is maximally general and may therefore be easily extended to junctions connecting more than two wires. The 1-2-symmetric three wire junction ("Y-junction") has been considered by us in Refs. [10-12, 15]. Work on the four-wire junction is in progress.

\section{Acknowledgements}

This paper is dedicated to the memory of the late Yehoshua Levinson. One of us (PW) has been fortunate to experience several years of collaboration and most enjoyable visits with him.

We thank D.G. Polyakov for helpful comments on the manuscript and A.P. Dmitriev, I.V. Gornyi and V.Yu. Kachorovskii for useful discussions. The work of D.A. was supported by the German-Israeli-Foundation (GIF), the Dynasty foundation, and a RFBR grant. The work of D.A. and P.W. was supported by the DFGCenter for Functional Nanostructures.

\section{References}

[1] T. Giamarchi, Quantum Physics in One Dimension (Clarendon Press, Oxford, 2003)

[2] A.O. Gogolin, A.A. Nersesyan, and A.M. Tsvelik, Bosonization and Strongly Correlated Systems (Cambridge University Press, Cambridge, 1998).

[3] C.L. Kane and M.P.A. Fisher, Phys. Rev. B 46, 15233 (1992), http://dx.doi.org/10.1103/PhysRevB.46.15233

[4] A. Furusaki and N. Nagaosa, Phys. Rev. B 47, 4631 (1993), http://dx.doi.org/10.1103/PhysRevB.47.4631

[5] I. Safi and H.J. Schulz, Phys. Rev. B 59, 3040 (1999), http://dx.doi.org/10.1103/PhysRevB.59.3040

[6] U. Weiss, R. Egger, and M. Sassetti, Phys. Rev. B 52, 16707 (1995), http://dx.doi.org/10.1103/PhysRevB.52.16707

[7] P. Fendley, A.W.W. Ludwig, and H. Saleur, Phys. Rev. B 52, 8934 (1995), http://dx.doi.org/10.1103/PhysRevB.52.8934

[8] D. Yue, L.I. Glazman, and K.A. Matveev, Phys. Rev. B 49, 1966 (1994), http://dx.doi.org/10.1103/PhysRevB.49.1966 
[9] D.N. Aristov and P. Wölfle, Phys. Rev. B 80, 045109 (2009), http://dx.doi.org/10.1103/PhysRevB.80.045109

[10] D.N. Aristov, A.P. Dmitriev, I.V. Gornyi, V.Y. Kachorovskii, D.G. Polyakov, and P. Wölfle, Phys. Rev. Lett. 105, 266404 (2010), http://dx.doi.org/10.1103/PhysRevLett.105.266404

[11] D.N. Aristov and P. Wölfle, Phys. Rev. B 84, 155426 (2011), http://dx.doi.org/10.1103/PhysRevB.84.155426
[12] D.N. Aristov and P. Wölfle, arXiv:1110.1159

[13] A. Furusaki and N. Nagaosa, Phys. Rev. B 54, R5239 (1996), http://dx.doi.org/10.1103/PhysRevB.54.R5239

[14] C. de C. Chamon and E. Fradkin, Phys. Rev. B 56, 2012 (1997), http://dx.doi.org/10.1103/PhysRevB.56.2012

[15] D.N. Aristov, Phys. Rev. B 83, 115446 (2011). http://dx.doi.org/10.1103/PhysRevB.83.115446 